\title{
A Burnett Apparatus for the Accurate Determination of Gas Compressibility Factors and Second Virial Coefficients, and an Evaluation of Its Capability Based on Some Results for Argon and Carbon Dioxide
}

\author{
Meyer Waxman and John R. Hastings \\ Institute for Basic Standards, National Bureau of Standards, Washington, D.C. 20234
}

(June 2, 1971)

\begin{abstract}
A Burnett apparatus has been developed for the determination of the compressibility factor of gases, including corrosive halogenic gases, to an accuracy of 0.1 percent to 0.01 percent at temperatures from 0 to $225^{\circ} \mathrm{C}$ and for pressures up to 250 bars. The apparatus has been used throughout this temperature and pressure range and at temperatures within $15^{\circ} \mathrm{C}$ of the critical temperature of the sample gas. The apparatus features a rugged yet highly sensitive and reproducible null-type pressure transducer to isolate the sample gas without introducing a pressure uncertainty greater than the precision of the pressure measurements, which is better than 1 part in 50,000. The pressure measurements are accurate to at least 1 part in 20,000 . The reduction of the data is discussed extensively. The capability of the apparatus has been evaluated in terms of the compressibility factor represented by a finite pressure or density virial expansion and derived from argon data at $25^{\circ} \mathrm{C}$ and pressures up to 250 bars and in terms of the second virial coefficient of carbon dioxide based on data at $50{ }^{\circ} \mathrm{C}$ and pressures below 35 bars. The value obtained for this second virial coefficient, $-102.2 \pm 0.2 \mathrm{~cm}^{3} / \mathrm{mol}$, compares favorably with the value of $-102.5 \mathrm{~cm}^{3} / \mathrm{mol}$ obtained from a recent correlation and is in substantial disagreement with the value of $-100.7 \pm 0.4 \mathrm{~cm}^{3} / \mathrm{mol}$ determined by Dadson and co-workers from the Burnett method and the piezometer method.
\end{abstract}

Key words: Adsorption, gas on metal; argon, compressibility factor of; Burnett data reduction; carbon dioxide, second virial coefficient of; compressibility factor; gas, compressibility factor of; gas, PVT properties of; measurements, gas; second virial coefficient.

\section{Introduction}

The accurate determination of the equation of state of a gas over a wide range of temperature and pressure provides important information about the gas both from a fundamental and an applied point of view. For example, the thermodynamic properties of a gas can be reliably extended beyond its ideal gas properties if its equation of state is known with sufficient accuracy. Equation of state data are conveniently represented by means of the compressibility factor. The compressibility factor $Z$ is defined in terms of the gas constant $R$ and the measurable gas variables pressure $P$, volume $V$, absolute temperature $T$, and quantity, expressed as the number of moles $n$, by the relationship

$$
Z=P V \mid n R T \text {. }
$$

The accuracy of $Z$ obtained from a straightforward measurement of each variable is limited by the accuracy with which the difficult measurements of $n$ and $V$ can be made. The direct or indirect measure- ment of one $(n)$ or both ( $n$ and $V$ ) of the difficult variables can be eliminated by the use of the virial expansions of $Z$ as a function of $P$ or of the density $\rho$ and the fact that the dependence of $Z$ on $P$ is dominantly linear at sufficiently low pressures and approaches the ideal gas law

$$
Z=P V / n R T=1
$$

in the limit as $P$ goes to zero $[1,2] .{ }^{1}$ The Burnett $P V T$ method [3-7] is an experimentally simplified method in which neither $n$ nor $V$ is measured; instead, accurate isothermal pressure measurements are made before and after stepwise expansions between two volumes. The decrease in the overall measurement error achieved by the elimination of $n$ and $V$ measurements can in principle lead to more accurate values of $Z$ than can be obtained from a method requiring the measurement of each variable in eq (1). For this reason we have developed a Burnett apparatus for the accurate determination of the compressibility factor of gases,

\footnotetext{
${ }^{1}$ Figures in brackets indicate the literature references at the end of this paper.
} 
including corrosive halogenic gases [8], at temperatures from 0 to $225^{\circ} \mathrm{C}$ and for pressures up to 250 bars $\left(1 \mathrm{bar} \equiv 10^{5} \mathrm{~N} \cdot \mathrm{m}^{-2}\right.$ ). The apparatus has been used by us throughout this temperature and pressure range including temperatures within $15^{\circ} \mathrm{C}$ of the critical temperature of the sample gas. The apparatus is equally well-suited and has been used for the accurate determination of second virial coefficients.

This paper is concerned with both the apparatus for obtaining Burnett data and the methods for reducing the data. These subjects are described briefly in the remainder of this section. In the next section the apparatus is discussed in more detail, and its distinctive features are emphasized. Since the reduction of the data obtained with the apparatus is an essential element in the evaluation of its capability, the data reduction is discussed extensively in the following section. The discussion is illustrated with results for the compressibility factor of argon at $25^{\circ} \mathrm{C}$ and pressures up to 250 bars and for the second virial coefficient of carbon dioxide at $50^{\circ} \mathrm{C}$.

The apparatus, shown schematically in figure 1 , consists essentially of a sample volume $V_{\mathrm{I}}$ and an expansion volume $V_{\text {II }}$ as well as components for (1) isolating, evacuating, or filling both volumes, (2) measuring the pressure in the sample volume, and (3) thermostating and measuring the temperature of the apparatus. The gas in the sample volume is isolated from the expansion volume by an expansion valve and from the external system by a metal diaphragm which is the pressure-responsive element of a null-type transducer. A counterbalancing gas acts on the other side of the diaphragm; the diaphragm is in its null position when the pressure on each side of the dia- phragm is the same. The pressure of the sample gas is obtained indirectly from the measurement of the pressure of the counterbalancing gas with a piston gage and a barometer. The sample and the expansion volumes include the appropriate volumes of the transducer, expansion and filling-evacuation valves, and the interconnecting pressure lines; in our apparatus all these volumes are thermostated at a temperature which is regulated to about $0.001{ }^{\circ} \mathrm{C}$ and measured with a platinum resistance thermometer. Because no correction needs to be made for temperature differences among these volumes, a correction that is often a significant source of error in PVT measurements is thereby eliminated. The transducer produces negligible variations of the sample volume and has a negligible effect on the high precision and accuracy of the pressure measurements. This is due to the excellent reproducibility of the null position of the diaphragm and the high sensitivity of the transducer.

The experimental procedure is begun by filling the sample volume to the initial pressure, thermostating the apparatus at the isotherm temperature $T_{c}$, isolating the volumes from each other, and then measuring the gas pressure $P_{0}$ in the sample volume. The corresponding compressibility factor $Z_{0}$ is

$$
Z_{0}=P_{0} V_{\mathrm{I}}\left(P_{0}\right) / n_{0} R T_{c},
$$

where $n_{0}$ represents the initial number of moles and $V_{\mathrm{I}}\left(P_{0}\right)$ indicates that the sample volume is pressure dependent as a result of its distortion under pressure.

In the next step, which we call the expansion step, the expansion volume is evacuated and isolated, and the gas in the sample volume is allowed to expand

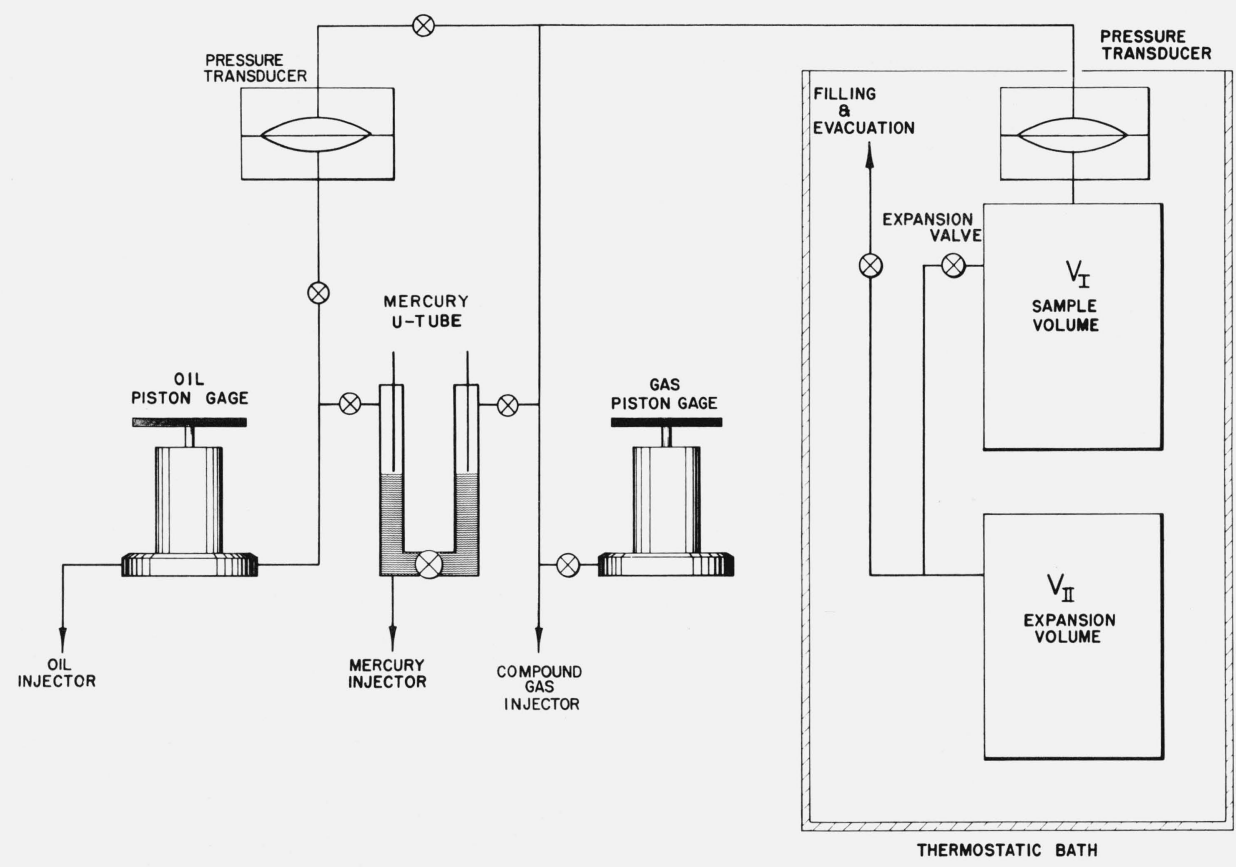

Figure 1. Schematic diagram of the Burnett PVT apparatus. 
into it. After temperature and pressure equilibration has occurred, the pressure $P_{1}$ of the gas that now occupies both volumes is measured. For this first expansion step the compressibility factor $Z_{1}$ is

$$
Z_{1}=P_{1}\left[V_{\mathrm{I}}\left(P_{1}\right)+V_{\mathrm{II}}\left(P_{1}\right)\right] / n_{0} R T_{c},
$$

where $n_{0}$ and $T_{c}$ are the same as in eq (3). By taking the ratio of eqs (3) and (4) and defining $N_{1}$ as the ratio of the combined sample and expansion volumes to the sample volume, i.e., $N_{1}=\left[V_{\mathrm{I}}\left(P_{1}\right)+V_{\mathrm{II}}\left(P_{1}\right)\right] / V_{\mathrm{I}}\left(P_{0}\right)$, one obtains

$$
P_{0} / P_{1}=N_{1} Z_{0} / Z_{1}
$$

in which $N_{1}$ depends slightly on both $P_{0}$ and $P_{1}$. The ratio of the volumes can be written as $N_{1}=N\left[1+\Delta N_{1}\left(P_{0}, P_{1}\right)\right]$, where $N$, known as the cell constant, is the value of the volume ratio in the limit as the pressure goes to zero and $\Delta N_{1}\left(P_{0}, P_{1}\right)$ is a cell constant correction for the dependence of the cell volumes on pressure.

Generally the sample volume is isolated again and the expansion step is repeated for the reduced quantity of gas $n_{1}$ that is now in the sample volume. This leads to a new pressure ratio, corresponding to eq (5),

$$
P_{1} / P_{2}=N_{2} Z_{1} / Z_{2},
$$

where $N_{2}=N\left[1+\Delta N_{2}\left(P_{1}, P_{2}\right)\right]$. This procedure is usually repeated to successively lower pressures with the successively smaller quantities of gas that remain in the sample volume after each expansion until the lowest pressures that can be measured both accurately and precisely are reached. The generalized form of eqs (5) and (6) for the $r$ th expansion is

$$
P_{r-1} / P_{r}=N_{r} Z_{r-1} / Z_{r}
$$

The combination of eq (7) and the relationship $P=\rho Z R T$, obtained from eq (1), leads to a corresponding Burnett equation in terms of the density

$$
\rho_{r}=\rho_{r-1} / N_{r}
$$

Equations (7) and (8) are basic equations in the reduction of Burnett data and may be transformed into different relationships in which the compressibility factor is represented by a finite virial expansion in either pressure or density. Any one of the relationships may be applied to Burnett data to form an overdetermined set of equations for evaluating the virial coefficients and generally the cell constant by means of nonlinear statistics [9]. Regardless of the relationship used, the cell constant is not determined from volume measurements. In the data reduction the pressure corrections $\Delta N_{1}, \Delta N_{2}, \ldots$ are not considered as unknown quantities but are estimated from theoretical and empirical distortion equations for pressure vessels or are determined from a separate distortion experiment.

\section{Apparatus and Experimental Procedure}

\subsection{Burnett Vessel and Gas Manifold System}

An overall view of the apparatus is shown in figure 2. In the Burnett vessel, as shown in figure 3, the sample chamber, $0.022 \mathrm{~m}$ i.d. by $0.076 \mathrm{~m}$ long, and the expansion chamber, $0.022 \mathrm{~m}$ i.d. by $0.051 \mathrm{~m}$ long, are bored from opposite ends of $0.064 \mathrm{~m}$ o.d. nickel rod to within $0.051 \mathrm{~m}$ of each other. Nonrotating end closures are used for coupling to the transducer and to the fillingevacuation line. The top gland nut is split to accommodate the transducer coupling. The coupling to the expansion valve is made through $0.0032 \mathrm{~m}$ o.d. by $0.00080 \mathrm{~m}$ i.d. stainless steel capillary tubing silverbrazed into the sample chamber inlet. The coupling from the expansion chamber is made through similar capillary tubing silver-brazed into a $T$-connection which in turn is coupled to the expansion valve and to the filling-evacuation valve. A well for the platinum resistance thermometer is bored radially into the Burnett vessel between the sample and the expansion chambers.

Since halogenic gases react readily with most metals, particularly if a trace of moisture is present, the components exposed to the sample gas are fabricated from metals such as nickel and stainless steel that are resistant to corrosion. We have found that gold provides still better resistance to corrosion than nickel and stainless steel do, and have therefore gold-plated every surface exposed to the sample gas in the Burnett volumes except the surfaces in the Burnett valves. Extreme care is taken to keep the system free of moisture at all times. The sample gas is not reclaimed; if necessary, neutralizing columns are used for its disposal. Cold traps prevent corrosive gases from entering the vacuum system, which includes low and high vacuum pumps, a cold cathode vacuum gage, and several thermocouple vacuum gages. Commercial diaphragm compressors boost the gas pressure from the supply cylinder pressure to about 600 bars for storage in high pressure receivers.

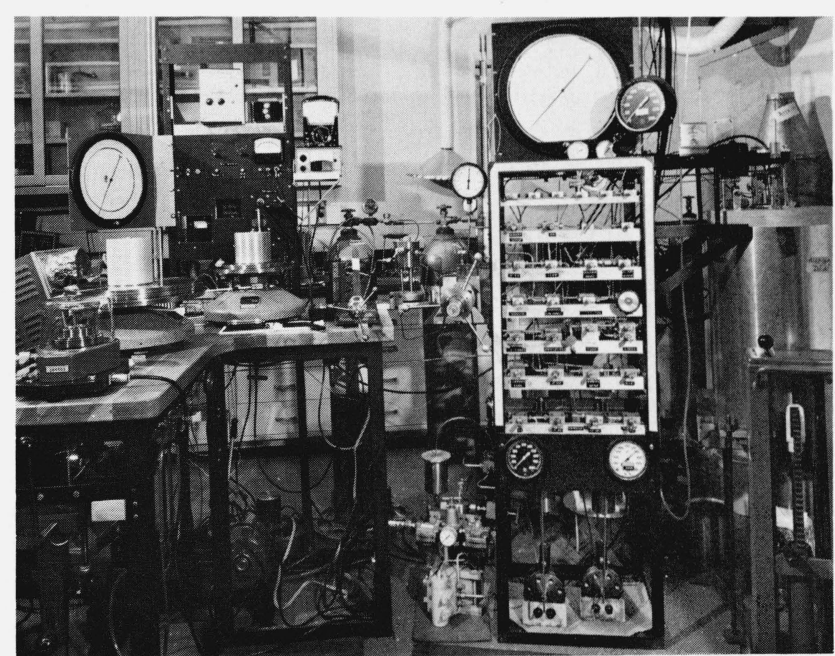

FigURE 2. Burnett PVT apparatus. 


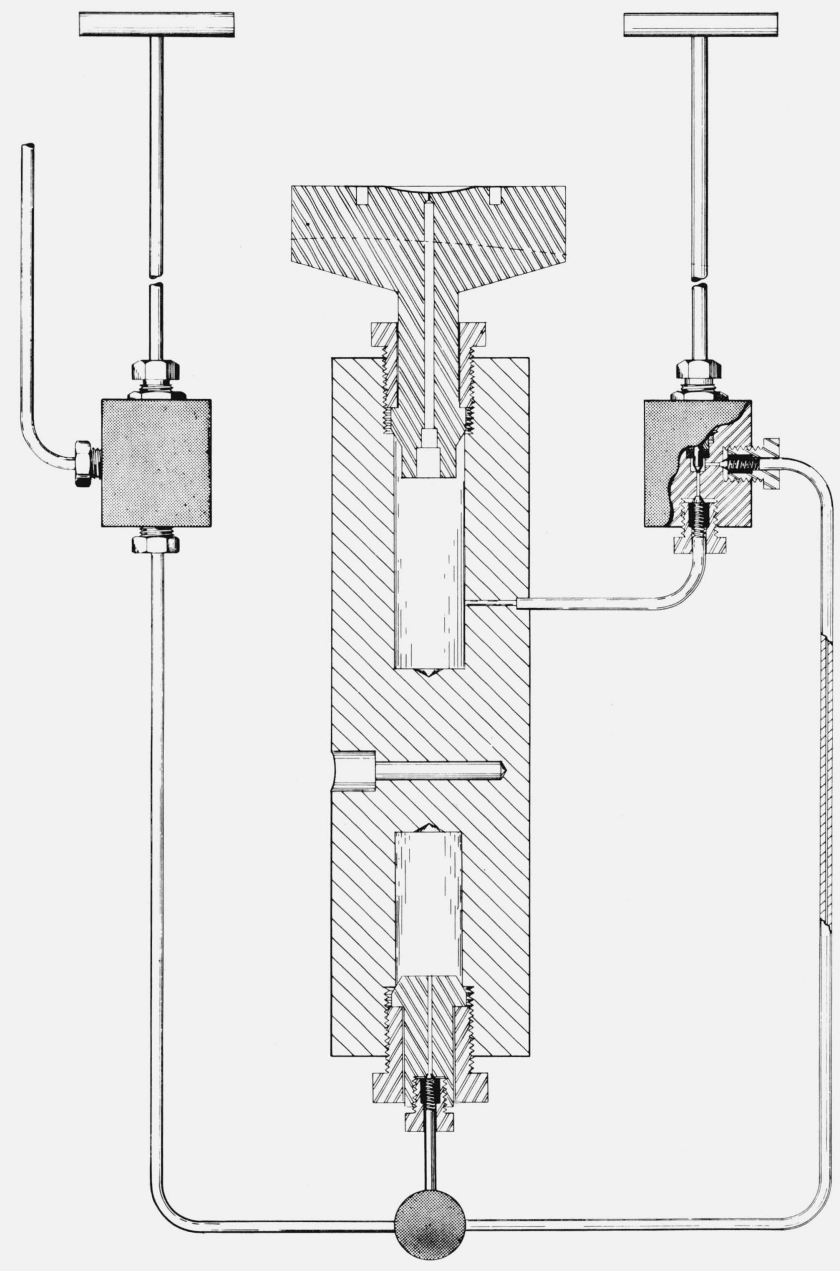

FigURE 3. Cross section of the Burnett volumes and valves.

Carefully selected commercial nonrotating-stem valves are used; our experience shows that for a gas system the sealing surfaces of rotating-stem valves tend to gall and consequently to leak. The condition of the expansion and the filling-evacuation valves is particularly important; minimum deformation of the valve seat, long periods of trouble-free use, and certainty of valve closure are essential characteristics. A small valve with a block of 316 stainless steel, ports to accommodate $0.0032 \mathrm{~m}$ o.d. capillary tubing, and a stem of 17-4 stainless steel hardened to 40 $\mathrm{R} / \mathrm{C}$ is used for each of these two valves. Valve blocks for all the valves are selected on the basis of the concentricity of the seat and the stem-packing section. The shoulder of the valve seat is beveled slightly and carefully lapped to the same angle as that of the conical end of the valve stem. The sealing surface is kept small to minimize the torque required for valve closure. To prolong valve life, all valves are closed with a torque wrench, and the required torque is recorded on each valve block.

\subsection{Pressure Measurement System}

\section{a. Transducer}

A rugged yet highly sensitive and reproducible null-type pressure transducer separates the sample gas from the pressure measurement system. The transducer is shown in figure 4; an earlier model has been described previously [10]. The transducer consists basically of a prestressed metal diaphragm as the pressure responsive element, a capacitance sensor for detecting deflections of the diaphragm as small as $0.025 \mu \mathrm{m}$ or less, and diaphragm backing surfaces incorporated as part of both the base and the capacitance sensor.

The diaphragm is plane and circular, $25 \mu \mathrm{m}$ thick, and prestressed to reduce inelastic behavior. The backing surfaces, spherically concave with a chord of $0.0238 \mathrm{~m}$ that determines the effective diameter of the diaphragm and a sagitta of $25 \mu \mathrm{m}$, are necessary because the stepwise expansions in the Burnett method result in temporary pressure imbalances across the diaphragm many times greater than the elastic range of a diaphragm with the required sensitivity.

The earlier model has been modified to improve its performance, especially above $125^{\circ} \mathrm{C}$. A metallized ceramic disk replaces the former metal-epoxy capacitance sensor assembly. This has enabled us to operate the apparatus at temperatures up to $225^{\circ} \mathrm{C}$, and the disk could even be used at temperatures as high as 500 or $600{ }^{\circ} \mathrm{C}$. The diaphragm is now fabricated from rolled nickel, instead of Monel, sheet stock; its thermal expansion thus closely matches that of the nickel base, and the diaphragm tension does not depend significantly on the temperature. A polytetrafluoroethylene ring, prestressed by the clamping of the top and the base of the transducer and supported by inner and outer metal extrusion rings, replaces the former $V$-ring as the outer pressure seal. The diaphragm sealing ring has been redesigned so that it stretches the diaphragm radially as it forms a pressure seal and thus does not distort the diaphragm when the transducer is assembled. Consequently the sample volume is more precisely defined and the transducer behaves more reproducibly. An important source of error is thereby reduced significantly.

The transducer capacitance, consisting of the capacitance contributed by the capacitor plate, diaphragm, and intervening gas plus the residual capacitance, constitutes one arm of a capacitance bridge. For each temperature and pressure the bridge is initially adjusted so that it is balanced when the pressure on each side of the diaphragm is the same. A subsequent pressure imbalance deflects the diaphragm from its null position and causes a bridge imbalance. The corresponding signal is amplified, phase detected, and indicated on a $100-0-100 \mu \mathrm{A}$ output meter. The counterbalancing gas pressure is then adjusted until the diaphragm returns to its null position as indicated by the bridge balance. 


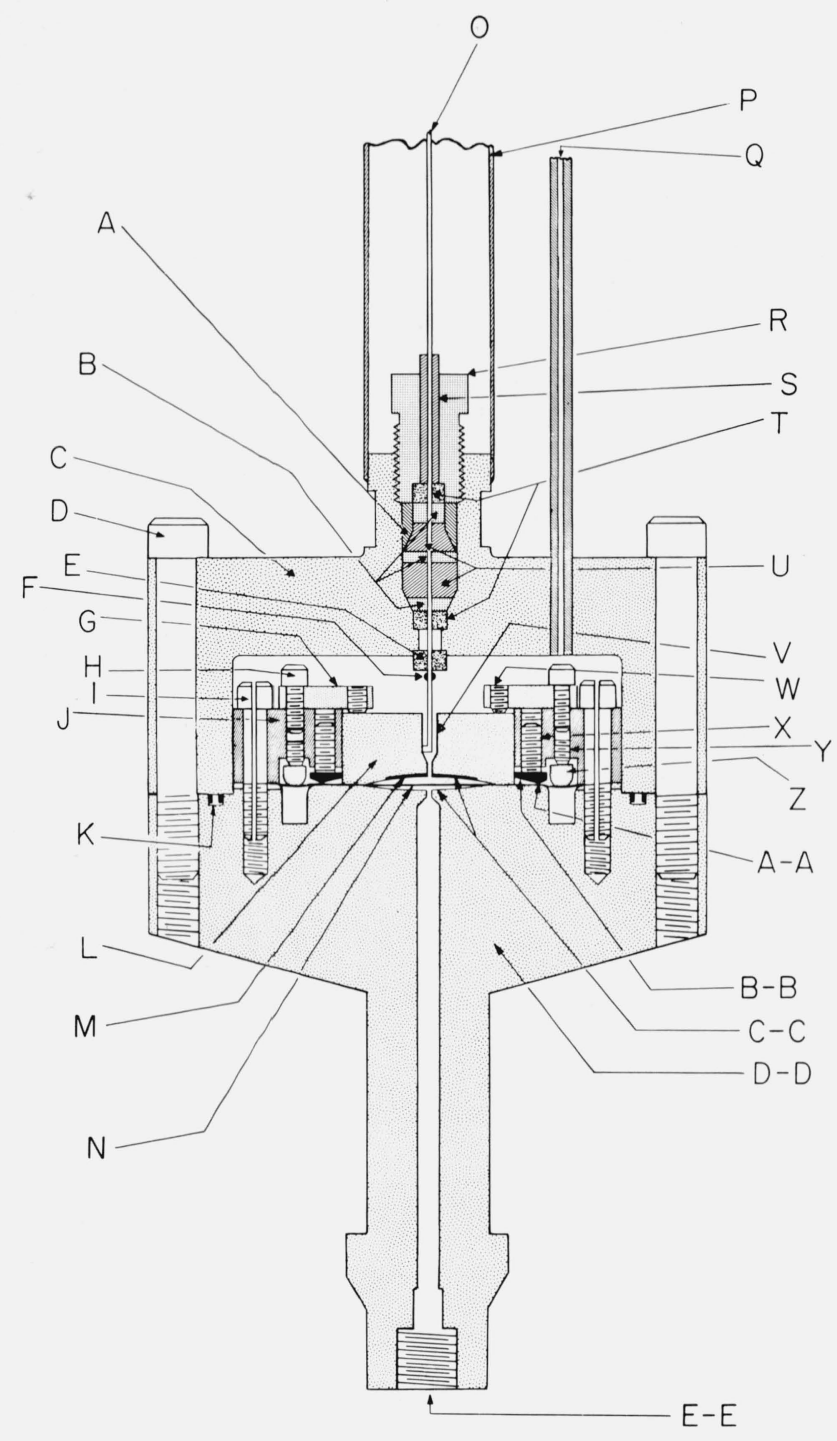

FigURE 4. Cross section of null-type pressure transducer.

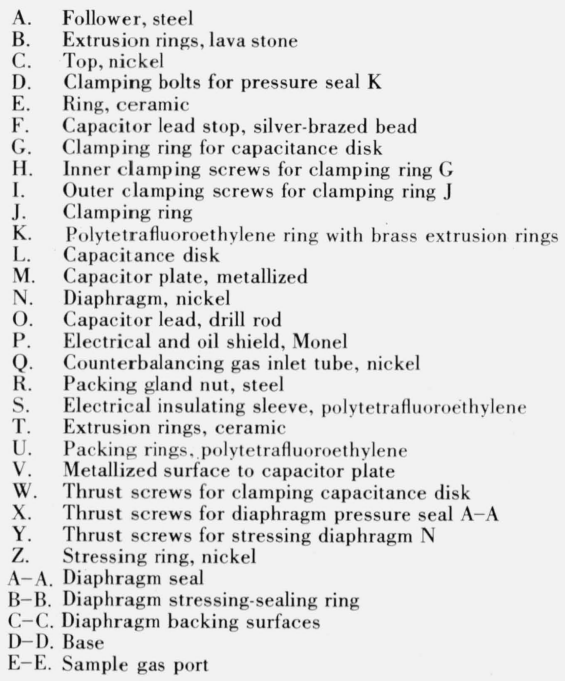

The temperature dependence of the transducer null capacitance is negligible for temperature changes even two orders of magnitude greater than the fluctuations of $0.001{ }^{\circ} \mathrm{C}$ about the isotherm temperature that represent the resolution of the temperature control system. For each isotherm the transducer is calibrated before Burnett measurements are made: The counterbalancing gas, which has been argon in all of our work, is admitted to both sides of the diaphragm, and the null capacitance is measured as a function of pressure from atmospheric pressure up to the highest Burnett pressure to be measured. The pressure dependence found does not represent any significant change in the diaphragm null position but is caused primarily by the change in the dielectric constant of the counterbalancing gas. The pressure sensitivity of the transducer, as used by us in the Burnett apparatus for pressures up to 250 bars, exceeds the sensitivity of the piston gages and the barometer used to measure the pressure.

\section{b. Piston Gages and Barometer}

Although Bourdon gages are used, for convenience, as pressure indicators at various locations in the apparatus, the basic pressure measurements are made with piston gages and a standard barometer-instruments which are primary pressure standards. Two controlled-clearance piston gages with an accuracy of better than 1 part in 20,000 cover the range from about 2 to 3 bars gage pressure up to 250 bars and beyond. A gage with gas as the pressure fluid covers the lower part of the range, and a gage with oil as the pressure fluid covers the higher part. Both gages cover the region from about 30 to 45 bars and in this overlap region they agree with each other to within 1 part in 40,000 to 50,000 of the measured pressure.

The nominal effective area of the oil gage piston is $26 \times 10^{-6} \mathrm{~m}^{2}$ and of the gas gage piston, about $340 \times 10^{-6} \mathrm{~m}^{2}$. The pistons are fabricated from tungsten carbide; the cylinders, from stainless steel. The top of the oil gage piston is hemispherical and fits into a conical recess in the coupling that connects the piston to the weight holder on which the weights are loaded. In the gas gage a lubricated ball bearing fits into a conical recess in both the piston and the coupling. In each case the purpose of the spherical contact, which represents our modification of the gages, is to reduce the amount of side thrust applied to the piston. Similarly, the weight holders and the weights that nest on them are machined carefully to avoid eccentric loading. The prevention of side thrust is particularly important for the gas gage because its piston receives little lateral support. The gas gage piston and cylinder must also be kept scrupulously clean. For each gage the load applied to the piston is spun manually and continues to spin for up to an hour or more before it stops.

Even though the pistons and cylinders were carefully lapped and cleaned before they were delivered to us, we were able to improve the performance of the gages by using a simple motor drive assembly to rotate the piston and move it up and down within its cylinder 
for several hours while the piston and cylinder were flooded with mineral spirits. The resultant burnishing increased the spin time, and the flooding helped to remove any fine particles that might have been present. The burnishing procedure can be used to improve performance at any time, particularly if the cylinder has become slightly scratched.

A simple piston gage without the controlled-clearance capability is used to make less accurate measurements over the range from 2 to 3 bars gage pressure down to 0.2 bars absolute pressure or less; the absolute measurements are made under an evacuated bell jar. The gage has three stainless steel piston and cylinder assemblies with nominal effective areas of $800 \times 10^{-6} \mathrm{~m}^{2}, 80 \times 10^{-6} \mathrm{~m}^{2}$, and $8 \times 10^{-6} \mathrm{~m}^{2}$, and uses gas as the pressure fluid.

The atmospheric pressure is measured with a standard barometer of modified U-tube design consisting of a column and a cistern. The level of the mercury in the column is detected photoelectrically and is measured with a 31 in $(0.79 \mathrm{~m})$ scale and a vernier graduated to 0.001 in $(25 \mu \mathrm{m})$; the level of the mercury in the cistern is detected electrically and is measured with a micrometer graduated to 0.0001 in $(2.5 \mu \mathrm{m})$. The back pressure above the mercury in the column is measured with a McLeod gage and is generally about $4 \times 10^{-6}$ bars or less. The accuracy of the barometer scale is easily checked with gage blocks. Repeated tests have shown that the barometric pressure measurements are accurate to $33 \times 10^{-6}$ bars.

\section{c. Other Components}

Small adjustments in the counterbalancing gas pressure are made with a compound gas injector whose internal volume is changed by the manual displacement of a $0.013 \mathrm{~m}$ or $0.0032 \mathrm{~m}$ diam piston. The counterbalancing pressure acts directly on the gas gages but is separated from the oil gage either by a commercial diaphragm transducer or by a mercury U-tube. The U-tube is an adaptation of an earlier model by Keyes [11] and is shown in figure 5. To reduce capillary effects, the lower part of each bore containing the mercury is made large in diameter. The bore diameter is reduced slightly above the mercury to make the gas volume in the bore a small fraction of the total volume of the counterbalancing gas. As a result, the bore gas volume has only a small effect on the performance of the gas injector. The mercury level in each column is indicated by electrical contact between the mercury and a fine metal probe whose height is initially adjusted to within $25 \mu \mathrm{m}$ of the reference height and is checked periodically. The modified design permits the valve at the base of the U-tube to be replaced conveniently.

\subsection{Temperature Control and Measurement System}

An oil bath and a servo heater system are used to control the temperature of the Burnett apparatus. As shown in figure 6 , the Burnett vessel, the transducer, the expansion and the filling-evacuation valves, and the interconnecting lines are all immersed in oil in an inner chamber located in a thermally insulated bath. The inner chamber is double-walled, and the space between the walls can be evacuated to reduce radial heat flow. An oil-circulating pump at the bottom of the bath is coupled mechanically to a motor mounted on the top. Oil drawn into the manifold and the pump is ejected upward into the inner chamber and flows by the Burnett

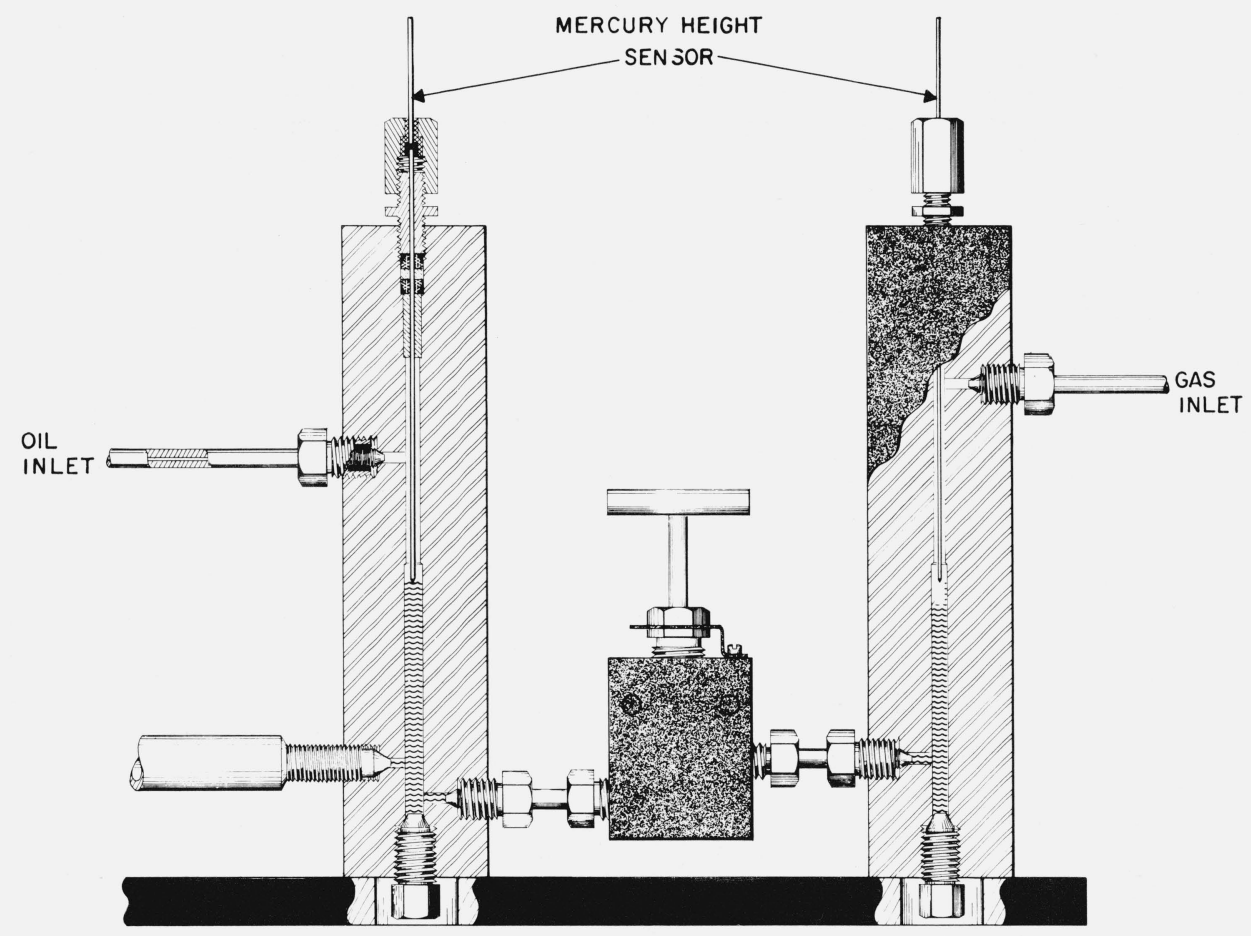

FigURE 5. Mercury U-tube. 


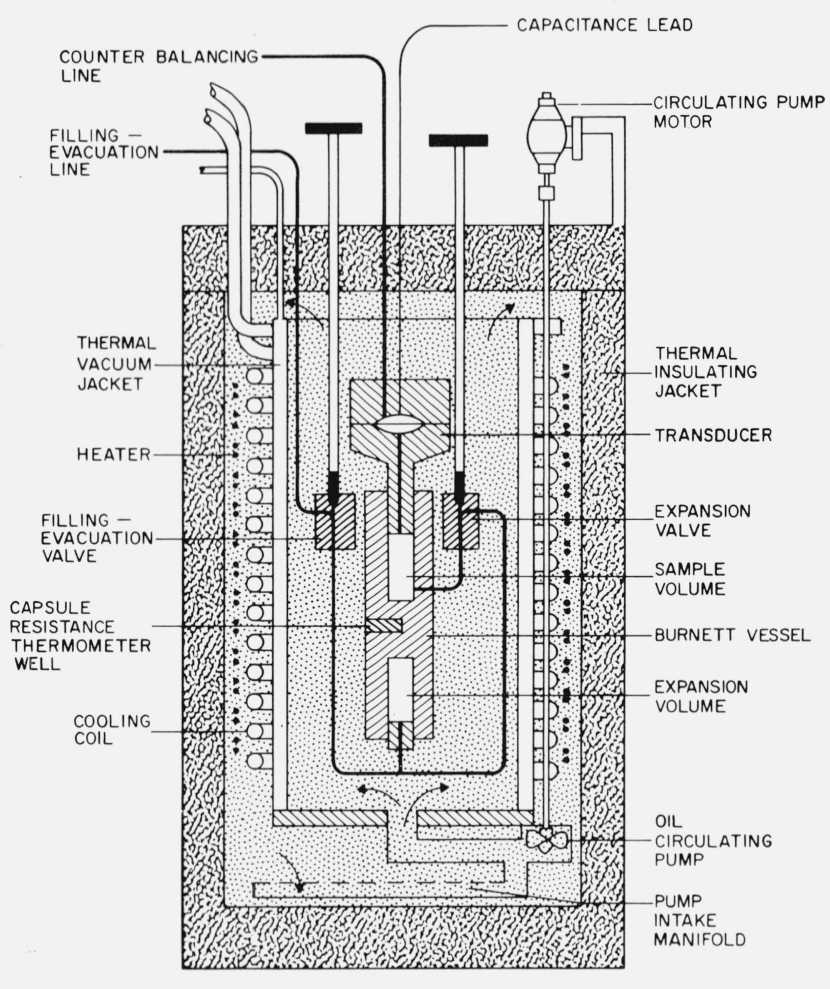

Figure 6. Oil bath.

apparatus, over the top of the inner chamber, and then downward past the heat exchanger to complete the cycle. Sufficient mixing of the oil occurs in the manifold and the pump to eliminate temperature gradients acquired during the flow of the oil past the heat exchanger.

The heat exchanger consists of a cooling coil and a series-parallel heater for low and high power output. A coolant is circulated through the coil and a refrigerated bath, which is thermostated to within $0.003{ }^{\circ} \mathrm{C}$ from -20 to $5{ }^{\circ} \mathrm{C}$. The cooling system is used for oil bath temperatures from $0{ }^{\circ} \mathrm{C}$ to slightly above room temperature.

The servo heater system consists of an adjustable Wheatstone bridge with a resistance thermometer constituting one arm, a d-c amplifier and recorder, a threemode controller for proportional, derivative, and integral response to the error signal, and a power amplifier connected to a heater circuit. The resolution of the temperature control is $0.001{ }^{\circ} \mathrm{C}$; slight differences from the desired temperature are readily corrected by the use of a variable resistor in the bridge circuit.

The temperature as defined by the International Practical Temperature Scale of 1968 is measured with a capsule platinum resistance thermometer located in the well between the sample and the expansion chambers in the Burnett vessel. The thermometer leads are brought out through a protection tube filled with oil to ensure good thermal contact between the thermometer and the Burnett vessel. A Mueller bridge with a photoelectric galvanometer as the detector is used to measure the resistance.
Differential five-junction copper-constantan thermocouples are used to measure temperature differences over the Burnett vessel and transducer as well as near the top of the oil bath. A small temperature difference can exist between the Burnett vessel and the top of the bath due to heat loss through the apparatus supports and oil-vapor loss to the surroundings. This difference is limited to $0.01{ }^{\circ} \mathrm{C}$ by means of a manually-controlled auxiliary heater at the top of the bath. Temperature differences over the Burnett vessel are less than $0.002{ }^{\circ} \mathrm{C}$. Electrical noise in the thermocouple circuits, which include the switching and measurement instrumentation, corresponds to a temperature uncertainty of less than $0.0001{ }^{\circ} \mathrm{C}$.

\subsection{Experimental Procedure}

An experimental procedure has been evolved which ensures the reproducibility of the pressure measurements, the precise definition of the sample volume, and the detection of any sample gas leakage. A series of runs is made at each isotherm temperature. Each run may include up to approximately eight expansions; the initial pressure for each run is chosen so as to give a well-distributed set of pressures for the isotherm. Before any Burnett measurements are made, the oil bath is thermostated at the desired temperature and the transducer is calibrated over the desired pressure range. The transducer null capacitance at atmospheric pressure is checked before and after each day's measurements.

The first step in a run is to evacuate the sample gas part of the apparatus and flush it thoroughly with the sample gas. After this part of the apparatus has been reevacuated, the sample and the expansion volumes are filled with fresh sample gas to the highest pressure desired for the run. In the flushing and the filling process and throughout the run, any pressure imbalance across the transducer diaphragm greater than a few bars is directed toward the base, which is more rugged than the capacitance sensor and its clamping arrangement and hence provides better diaphragm support. This precaution helps to ensure the stability of the diaphragm null position and thus aids in the precise definition of the sample volume. Similarly, as a pressure measurement is made, the diaphragm is slightly overloaded toward the base prior to the final balancing of the counterbalancing and the sample gas pressures.

After the filling process is completed and the fillingevacuation valve closed, sufficient time is allowed for temperature and pressure equilibration. During the course of a run the temperature of the Burnett vessel may vary over a range of about $0.002{ }^{\circ} \mathrm{C}$ due to instabilities primarily in the temperature control bridge; equilibration times are chosen to keep shortterm temperature changes well within this range and temperature differences within the ranges discussed earlier.

While the equilibration is taking place, preparations are made for the first set of Burnett measurements. The counterbalancing gas, which was maintained at a higher pressure than the sample gas during the filling 
process, is gradually vented until the transducer null indicator returns to its zero position. The approximate counterbalancing gas pressure is then read from a Bourdon gage, and a variable capacitor in the capacitance bridge is adjusted in accordance with the transducer calibration so that the bridge balance will truly correspond to the diaphragm null position. The piston gage to be used for the precise pressure measurement is loaded with approximately the right combination of weights that will be needed.

When the resistance thermometer and the differential thermocouple readings show that the equilibration is essentially complete, the expansion valve is closed to isolate the sample and the expansion volumes. While the valve is slowly closed, the diaphragm is maintained in its null position by adjustment of the counterbalancing gas pressure with the compound gas injector. An amplifier and supplementary null indicator about ten times as sensitive as the transducer output meter are used in this process, partly because of their greater sensitivity and also because the location of the supplementary null indicator is more convenient to the person closing the valve and operating the compound gas injector.

After the expansion valve is closed, the first set of Burnett measurements can be made. In the operation of the piston gage a precise zeroing of the transducer output meter would require an unnecessarily long time to be achieved. Instead the gage operator calibrates the output meter over its central range by noting the difference in the scale readings that correspond to two nearly equal loads on the gage. The slight difference in the diaphragm position represented by the different scale readings changes the sample volume, which is bounded by the diaphragm, by a negligible amount less than $2 \mathrm{ppm}$. The scale readings are made when the piston is at its reference height as shown by an electronic proximity sensor mounted underneath the weights loaded on the gage. The height of a gas gage piston is adjusted by means of the compound gas injector; the height of the oil gage piston is adjusted by means of a separate oil injector. For either type of gage the piston is raised above its reference height by means of the appropriate injector and is then allowed to fall slowly down to its reference height while the piston and its load are spinning steadily. The gage pressure and barometric pressure measurements are made almost simultaneously, and the resistance thermometer and differential thermocouple measurements are made immediately afterward. These measurements constitute the first set of Burnett measurements.

Next the expansion valve is opened and after about a minute is closed as before. A second set of Burnett measurements is made. Since the expansion step has not been performed, this second set of measurements should be in close agreement with the first set. If the second set, in fact, does not differ significantly from the first, the filling-evacuation valve is opened and the expansion volume is evacuated. After isothermal conditions are reestablished and a thermocouple vacuum gage, located as close to the expansion volume as is convenient, shows a pressure of about $4 \times 10^{-6}$ bars, a third set of measurements is made. If this set does not differ significantly from the first two, the fillingevacuation valve is closed and the expansion valve is opened. The gas initially in the sample volume expands to fill the expansion volume as well. After this first expansion the measurement procedure is repeated to successively lower pressures.

To be considered satisfactory, the three sets of measurements corresponding to the initial filling or a subsequent expansion must show a pressure reproducibility of 1 part in 50,000 or better. Occasionally a set of measurements will be repeated if some temporary disturbance, such as a temperature or barometric fluctuation, has caused a poorer reproducibility. If a serious experimental failure, such as sample gas leakage, were to occur, the measurements for that run would not be used in the data reduction.

\section{Data Reduction and Discussion of Results}

\subsection{Background}

In contrast to the simplicity of the experimental measurements, the reduction of Burnett data involves rather formidable nonlinear statistical analysis of relationships containing the compressibility factor, defined by a pressure or a density virial expansion, and optional constants. The authors have previously presented a general discussion of the analysis of different Burnett relationships using two nonlinear statistical methods, due to Gauss and to Deming [9]. Related papers have also been published by others $[12,13]$.

Our experience indicates that compressibility factors and second virial coefficients calculated from Burnett data of high precision and accuracy are as accurate as those calculated from the best data obtained with any other $P V T$ method. Various treatments of relationships, that is, various models, based on eqs (7) and (8) have been used in our analysis. In these models the cell constant, where it appears, is regarded as a single parameter common to all the experimental runs, as a separate parameter for each run, or as a constant to be determined from Burnett measurements for a nearly ideal gas. Meaningful comparisons of the results are difficult for at least two reasons: (1) the same systematic errors may propagate differently in each model and (2) the number of parameters to be evaluated varies from one model to another. The effect of the latter is subtle; it may introduce an analytical bias, as the magnitude of the correlation between the parameters, which is basically high, increases significantly with an increase in the number of parameters. It is not surprising, therefore, that slight discrepancies exist among the results of different models, even when applied to the same data. For example, a comparison of the compressibility factors derived from argon data taken with our Burnett apparatus shows discrepancies among the values of approximately 0.01 percent to 0.04 percent, which, 
although small, are nevertheless greater than the estimated statistical uncertainties of less than 0.01 percent. For the six models considered, the results of four show comparative discrepancies of about 0.01 percent. Another example of this type of discrepancy occurred in an informal round-robin analysis of helium data by participants at a two-day Burnett conference held at the National Bureau of Standards in February 1969. Initial analyses indicated discrepancies of 0.04 percent among the results of the different models as compared to statistical uncertainties of 0.01 percent in the results themselves.

We shall not repeat our previous detailed presentation of the nonlinear statistical analysis of Burnett data [9], but shall present a brief discussion which emphasizes the important differences between the various models in order to help one choose the models that introduce the least bias. The fact that the data can be reduced in several ways is an attractive feature of the Burnett method and enables one to study the different effects of systematic errors.

The basic Burnett equations may be transformed into different relationships among the variables of interest. In terms of a finite virial expansion in pressure,

$Z=1+\sum_{i=1}^{I} b_{i} P^{i}$, eq (7) may be expressed as

$$
\begin{gathered}
\frac{P_{r-1}}{P_{r}}=N_{r} \frac{\left(1+\sum_{i=1}^{I} b_{i} P_{r-1}^{i}\right)}{\left(1+\sum_{i=1}^{I} b_{i} P_{r}^{i}\right)}, \\
P_{r}\left(N_{1} N_{2} \ldots N_{r}\right)=\frac{P_{0}}{Z_{0}}\left(1+\sum_{i=1}^{I} b_{i} P_{r}^{i}\right),
\end{gathered}
$$

$\frac{P_{r-2} P_{r}}{P_{r-1}^{2}}=\frac{1+\Delta N_{r-1}\left(P_{r-2}, P_{r-1}\right)}{1+\Delta N_{r}\left(P_{r-1}, P_{r}\right)}$

$$
\times \frac{\left(1+\sum_{i=1}^{I} b_{i} P_{r-2}^{i}\right)\left(1+\sum_{i=1}^{I} b_{i} P_{r}^{i}\right)}{\left(1+\sum_{i=1}^{I} b_{i} P_{r-1}^{i}\right)^{2}} .
$$

In terms of a finite virial expansion in density,

$Z=1+\sum_{j=1}^{J} B_{j} \rho^{j}$, eq (8) may be expressed as

$P_{r}=\frac{\rho_{0}}{N_{1} N_{2} \ldots N_{r}} R T_{c}\left[1+\sum_{j=1}^{J} B_{j}\left(\frac{\rho_{0}}{N_{1} N_{2} \ldots N_{r}}\right)^{j}\right]$.

It should be noted that in these equations the second virial coefficient is represented by $b_{1}$ or $B_{1}$. For both the pressure and the density relationships

$$
N_{r}=N\left[1+\Delta N_{r}\left(P_{r-1}, P_{r}\right)\right]
$$

and

$$
\begin{aligned}
N_{1} N_{2} \ldots & N_{r}=N^{r}\left[1+\Delta N_{1}\left(P_{0}, P_{1}\right)\right] \\
& \times\left[1+\Delta N_{2}\left(P_{1}, P_{2}\right)\right] \ldots\left[1+\Delta N_{r}\left(P_{r-1}, P_{r}\right)\right] .
\end{aligned}
$$

Any one of these relationships may be applied to Burnett data to form an overdetermined set of equations for evaluating the virial coefficients and the other parameters. The latter consist of the cell constant (except in eq (11) where the cell constant has been eliminated), the initial density $\rho_{0}$ in eq (12), and sometimes the quantity $P_{0} / Z_{0}$ in eq (10). The latter two parameters are characteristic of an individual run. As mentioned in the introduction, the pressure corrections $\left(\Delta N_{1}, \Delta N_{2}, \ldots\right)$ are not considered as parameters but are determined independently of the parameter evaluation.

\subsection{Compressibility Factor of Argon at $25{ }^{\circ} \mathrm{C}$}

Compressibility factor results derived from the different relationships applied to our argon data are given in table 1 [9]. The models are designated briefly at the top of each column. For each model the parameters were evaluated simultaneously for all the data. Where a fixed cell constant was used, it was predetermined from eq (9) applied to helium data taken at the same temperature for pressures below 35 bars. For these conditions helium behaves nearly as an ideal gas, a characteristic which simplifies the data reduction.

The results at each pressure are essentially the same except for those derived from the density relationship with an adjustable cell constant included as either a separate parameter for each run or a single parameter common to all the runs. The discrepancies among the first four columns, which are about 0.01 percent, could be due to the presence of systematic error which propagates differently in each model. A likely source of such error is the correction for the volume distortion of the sample and expansion chambers with pressure. A study of simulated Burnett problems based on our argon data indicated that other probable sources of error had a negligible effect on the compressibility factor. In this study a simulated systematic error of 1 part in 30,000 was superimposed on the argon pressure data. The density reduction of these data, with the cell constant either fixed or regarded as a parameter, resulted in a change in the compressibility factor of less than 0.001 percent for all the pressures. An effect of less than 0.001 percent was also obtained when a simulated systematic error of 1 part in 20,000 was superimposed on the $R T$ product in a density reduction of the data.

The volume distortion correction was determined from distortion equations and strain gage measurements of the external deformation of the Burnett 
TABLE 1. Comparison of results derived from Burnett argon data at $25{ }^{\circ} \mathrm{C}$ and to 250 bars $\mathrm{a}^{\mathrm{a}, \mathrm{b}}$

3 Runs $(7,7, \& 6$ expansions/run)

\begin{tabular}{|c|c|c|c|c|c|c|}
\hline & \multicolumn{3}{|c|}{ Pressure series formulation } & \multicolumn{3}{|c|}{ Density series formulation } \\
\hline & $\begin{array}{l}\mathrm{Eq}(9) \\
\text { Cell constant } \\
\text { fixed }\end{array}$ & $\begin{array}{c}\mathrm{Eq}(9) \\
\text { One cell constant } \\
\text { adjusted }\end{array}$ & $\begin{array}{c}\text { Eq }(11) \\
\text { Cell constant } \\
\text { eliminated }\end{array}$ & $\begin{array}{l}\text { Eq }(12) \\
\text { Cell constant } \\
\text { fixed }\end{array}$ & $\begin{array}{l}\text { Eq (12) } \\
\text { One cell constant } \\
\text { adjusted }\end{array}$ & $\begin{array}{c}\mathrm{Eq}(12) \\
\text { All cell constants } \\
\text { adjusted }\end{array}$ \\
\hline $\mathrm{N}$ & 1.74505 & 1.74502 & & 1.74505 & 1.74511 & $\begin{array}{l}1.74511 \\
1.74509 \\
1.74511\end{array}$ \\
\hline $\begin{array}{l}Z_{10.133 \text { bars }} \\
Z_{101.33 \text { bars }} \\
Z_{202.65 \text { bars }}\end{array}$ & $\begin{array}{r}0.99384 \\
.95356 \\
.95202\end{array}$ & $\begin{array}{r}0.99387 \\
.95365 \\
.95209\end{array}$ & $\begin{array}{r}0.99388 \\
.95367 \\
.95212\end{array}$ & $\begin{array}{r}0.99380 \\
.95352 \\
.95194\end{array}$ & $\begin{array}{r}0.99375 \\
.95333 \\
.95169\end{array}$ & $\begin{array}{r}0.99376 \\
.95334 \\
.95173\end{array}$ \\
\hline
\end{tabular}

a The compressibility factor is shown to five digits to indicate the differences in the numerical results and not to indicate the overall accuracy.

b $\mathrm{l}$ bar $\equiv 10^{5} \mathrm{~N} \cdot \mathrm{m}^{-2}$.

vessel due to internal pressure $[14,15]$. The effect of the distortion correction and its estimated uncertainty on the compressibility factor is shown in figure 7 for argon data analyzed with the use of the pressure equation (10). The effect of the uncertainty is small, about 0.01 percent at the maximum pressure of 250 bars. The results of simulated problems indicate that the effect is the same in the density formulation. In the pressure series formulation the effect of the distortion correction uncertainty is consistent with the small differences between the results derived with the cell constant fixed and those derived with the cell constant evaluated or eliminated.

We now consider the basis for the differences, particularly at the higher pressures, which are significantly greater than 0.01 percent. These are between the results derived from the pressure series formulation and the results from the density series formulation, particularly with the cell constant included as either a separate parameter for each run or a single parameter common to all the runs. If the exact model were known, that is, if all the systematic errors

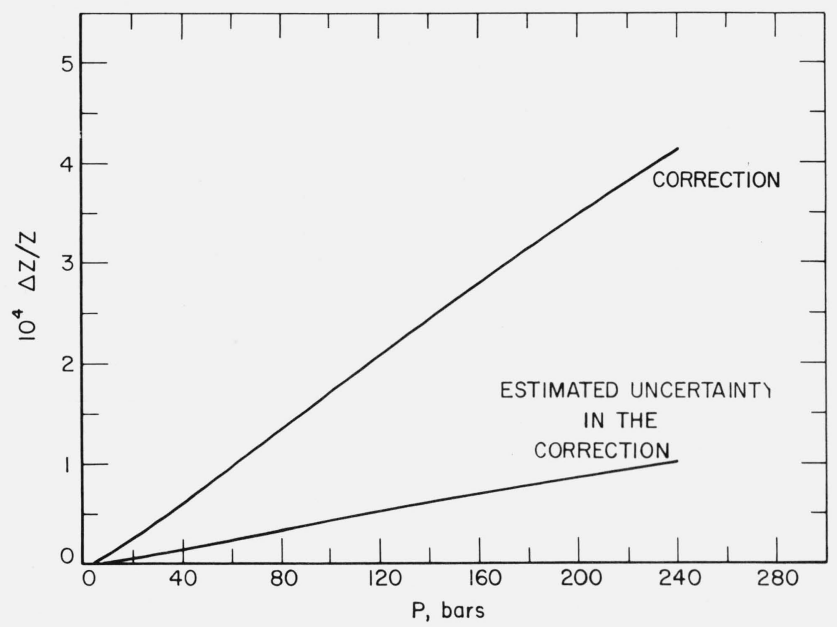

FigURE 7. Relative compressibility factor correction due to pressure distortion of Burnett cell volumes, argon run. were included in each equation, then the uncertainties in the values of the compressibility factor, the cell constant, and the initial densities would be consistent with the magnitude of any random errors in the pressure. This has been illustrated with a simulated Burnett problem for a particular model in which random errors were superimposed on the pressure data [16]. In the real world one cannot account exactly for all of the systematic errors. Consequently, a bias is introduced in the statistical analysis, and the results are not as reliable as the statistical uncertainties indicate. In addition, the number of parameters varies from one model to another. This variation adds to the bias since the absolute value of the correlation between the parameters increases significantly with an increase in the number of parameters.

In general the correlation between virial coefficients is high regardless of which $P V T$ method is used to determine them. In the Burnett method additional highly correlated parameters are also present. In the density reduction of the argon data with a fixed cell constant, the absolute value of the computed correlation between each of the virial coefficients is about 0.85 on a normalized basis of 0 to 1 , whereas it is about 0.6 between each of the initial densities and about 0.75 between any one of the virial coefficients and any one of the initial densities. In contrast, if the cell constant is included as a parameter, the statistical fit is improved over that for the fixed cell constant, in the sense that the sum of squares is reduced. However, the correlation between any two of the parameters increases significantly! The correlation between each of the virial coefficients becomes about 0.93 ; between each of the initial densities, about 0.994 ; and between any one of the virial coefficients and any one of the initial densities, about 0.87 . The correlation between the cell constant and any one of the virial coefficients is about 0.88 and between the cell constant and any one of the initial densities, about 0.995 . Exceptionally high correlations such as 0.994 and 0.995 indicate that these parameters are not truly independent and thus cannot be accurately evaluated [17]. 
Additional data, not highly correlated to the data already in the analysis, would reduce the high parameter correlation. As a result, the evaluation of the parameters would be enhanced and any analytical bias in the results would be reduced. In one approach the analysis would consist of data from the same apparatus for two or more sample gases with different compressibility characteristics. The data would be taken at the same temperature, and a single cell constant parameter, common to all the data, would be evaluated. Another approach would be to use a fixed cell constant obtained from helium data. This approach is less desirable because the cell constant would be entered without any statistical uncertainty, that is, as an exact quantity, and hence the computed statistical uncertainties would be too small. The significance of high parameter correlation should be considered carefully in estimating the reliability of results obtained from the density reduction methods. For the pressure reduction methods the situation is similar, although the parameter correlation is not as high.

Our values of the compressibility factor shown in table 2 represent the average of the results of the three pressure models and the density model with a fixed cell constant; the results of the density models with one or three separate cell constants were not included in the average because they were considered to be less reliable due to the high correlation between the parameters. For pressures up to 250 bars the overall accuracy of the averaged compressibility factor is estimated to be within \pm 0.0002 on the basis of our error analysis. The statistical uncertainty of each value of the compressibility factor is no greater than one sixth of the overall accuracy of the averaged compressibility factor. Table 2 also includes comparative values of the argon compressibility factor derived from the piezometer results of Michels [18] for pressures up to 202.7 bars. The maximum difference is small, only 0.05 percent at 202.7 bars; $P V T$ results are generally considered to be accurate if the overall error is less than 0.1 percent.

TABLE 2. Comparative values of the compressibility factor for argon at $25^{\circ} C^{\text {a }}$

\begin{tabular}{|c|c|c|}
\hline $\begin{array}{l}\text { Pressure } \\
\text { bars }\end{array}$ & $\begin{array}{l}\text { This paper }{ }^{\mathrm{b}} \\
\text { Compressibility factor }\end{array}$ & $\begin{array}{c}\text { Michels }^{c} \\
\text { Compressibility factor }\end{array}$ \\
\hline $\begin{array}{l}10.133 \\
101.33 \\
202.65\end{array}$ & $\begin{array}{r}0.9938 \\
.9536 \\
.9520\end{array}$ & $\begin{array}{r}0.9938 \\
.9534 \\
.9515\end{array}$ \\
\hline
\end{tabular}

a $1 \mathrm{bar} \equiv 10^{5} \mathrm{~N} \cdot \mathrm{m}^{-2}$

${ }^{b}$ The overall accuracy is estimated to be \pm 0.0002 .

$[18]$.

\subsection{Second Virial coefficient of Carbon Dioxide at $50{ }^{\circ} \mathrm{C}$}

In addition to accurate values of the compressibility factor, reliable second virial coefficients are also obtainable from the Burnett method. Our own experience with argon at $25^{\circ} \mathrm{C}$ and with carbon dioxide at $50{ }^{\circ} \mathrm{C}, 19$ degrees above its critical temperature, shows that this is true over a wide range of reduced temperatures greater than unity. ${ }^{2}$ The argon results are for a reduced temperature of 1.98 and have been discussed previously [9]; the carbon dioxide results, which are discussed in the following paragraphs, are new and are for a reduced temperature of 1.06.

Two groups of carbon dioxide measurements were made: one group over the pressure range from 35 to 4.5 bars and another group over the range from 4.5 to 0.5 bars. The higher pressure data are the more accurate data and were reduced by the density model with a fixed cell constant determined from helium data at the same temperature. From the more accurate data a second virial coefficient for carbon dioxide of $-102.25 \pm 0.04 \mathrm{~cm}^{3} / \mathrm{mol}$ was obtained, where the uncertainty is only the statistical uncertainty and is necessarily too small because it does not include the uncertainty in the cell constant. Our estimate of the overall systematic uncertainty, based on our error analysis, is $0.2 \mathrm{~cm}^{3} / \mathrm{mol}$. This value of the second virial coefficient was supported by a graphical evaluation which included the less accurate carbon dioxide data for the lower pressures. It compares favorably with the value of $-102.5 \mathrm{~cm}^{3} / \mathrm{mol}$ obtained from a recent correlation [19] which included an evaluation of all existing data but is in substantial disagreement with the value of $-100.7 \pm 0.4 \mathrm{~cm}^{3} / \mathrm{mol}$ determined by Dadson and his co-workers [20,21]. It should be noted that Dadson et al. carried out their measurements with both the Burnett method and the piezometer method. The fact that their results from both these methods agreed with each other and yet disagreed with the results of the correlation prompted our own measurements. Our results and more detailed comparisons will be the subject of a later publication.

Various potential sources of systematic error were investigated in our own work. In simulated problems based on the higher pressure data, superimposed systematic errors of \pm 25 percent in the distortion correction and 1 part in 20,000 in the $R T$ product had only a negligible effect on the second virial coefficient. The uncertainty in the distortion correction would be expected to have a negligible effect since the distortion correction itself is very small even at the highest pressures in the carbon dioxide data. A cell constant was computed for the carbon dioxide data in a separate calculation by treating it as an adjustable parameter in the density series formulation. The value obtained agreed with the predetermined value obtained from helium data to within 1 part in 20,000 , which is excellent agreement in view of the large magnitude of the nonideality of carbon dioxide at $50{ }^{\circ} \mathrm{C}\left(T / T_{\text {critical }}=1.06\right)$ and of the extremely high correlation between the parameters in the analysis.

The possibility of adsorption of carbon dioxide on the surfaces of the sample and the expansion volumes was investigated by means of a graph of the low pressure data in which the pressure ratio $P_{r-1} / P_{r}$ was plotted

\footnotetext{
2 The reduced temperature is the ratio of the sample gas temperature to its critical temperature, where both these temperatures are expressed on the absolute scale.
} 
as a function of the pressure $P_{r-1}$. The significance of the graph is based on the equation

$$
\frac{P_{r-1}}{P_{r}}=N+b_{1}(N-1) P_{r-1},
$$

which is the low pressure form of eq (9). ${ }^{3}$ The extrapolation of the curve drawn through the points to the vertical axis yielded an intercept whose value, the cell constant, was essentially the same as the predetermined value obtained from the helium data. This agreement would not be expected if adsorption were present. For a nonpolar gas such as carbon dioxide and for our range of pressures, adsorption is expected to be saturated [22]; that is, above a relatively low pressure very little more adsorption occurs as the pressure is increased further. In the sample volume the adsorbed gas would remain essentially unchanged upon an expansion, whereas in the expansion volume gas would be desorbed in the evacuation process and adsorbed in the expansion process. As a result, the number of free molecules would be reduced upon an expansion, and the ratio $P_{r-1} / P_{r}$ would therefore increase rapidly at low pressures. The extrapolation to zero pressure would yield a value for the cell constant that is greater than the value predetermined from helium data for which adsorption is not significant. Furthermore, the graph of this ratio versus pressure would show increasing nonlinearity at low pressures instead of the decreasing nonlinearity characteristic of real gases as the effect of the third and higher order virial coefficients not shown in eq (13) becomes insignificant. Neither of these signs of adsorption is present in our data. Hence we feel confident that any adsorption in our apparatus is so slight as to have an insignificant effect on our results.

We gratefully acknowledge the benefit we have received from general discussions with $\mathrm{M}$. Klein and from comments regarding adsorption made by P. T. Eubank.

\section{References}

[1] Mason, E. A., and Spurling, T. H., The Virial Equation of State (Pergamon Press, Oxford, 1969).

[2] Levelt Sengers, J. M. H., The experimental determination of the equation-of-state of gases and liquids at low temperatures, Physics of High Pressures and the Condensed Phase, Ed. A. van Itterbeek, pp. 60-97 (North-Holland Publishing Co., Amsterdam, 1965).

\footnotetext{
${ }^{3}$ Equation (13), together with the equation $B_{1}=R T b_{1}$, was used in the graphical evaluation of the second virial coefficient.
}

[3] Burnett, E. S., Compressibility determinations without volume measurements, J. Appl. Mech. 3, A136-A140 (1936).

[4] Pfefferle, W. C., Jr., Goff, J. A., and Miller, J. G., Compressibility of gases. I. The Burnett method. An improved method of treatment of the data. Extension of the method to gas mixtures, J. Chem. Phys. 23, 509-513 (1955).

[5] Liley, P. E., Thermodynamic Properties of Steam/Carbon Dioxide Mixtures at High Temperature and Pressure, Thesis, University of London (1957).

[6] Silberberg, I. H., Kobe, K. A., and McKetta, J. J., Gas compressibilities with the Burnett apparatus, J. Chem. Eng. Data 4, 314-330 (1959).

[7] Hoover, A. E., Canfield, F. B., Kobayashi, R., and Leland, T. W.. Jr., Determination of virial coefficients by the Burnett method, J. Chem. Eng. Data 9, 568-573 (1964).

[8] Waxman, M., Hilsenrath, J., and Chen, W. T., The compressibility factor and the second virial coefficient of boron trifluoride from 0 to $225^{\circ} \mathrm{C}$ and for pressures up to 250 bars, (to be published).

[9] Waxman, M., Hastings, J. R., and Chen, W. T., Nonlinear statistical analysis of Burnett PVT data, Proceedings of the Fifth Symposium on Thermophysical Properties, Ed. C. F. Bonilla, pp. 248-261 (ASME, New York, 1970).

[10] Waxman, M., and Chen, W. T., A rugged null-type pressure transducer of high reproducibility for accurate gas phase PVT measurements, J. Res. Nat. Bur. Stand. (U.S.), 69C (Eng. and Instr.), No. 1, 27-33 (1965).

[11] Keyes, F. G., Methods and procedures used in the Massachusetts Institute of Technology program of investigation of the pressures and volumes of water to $460{ }^{\circ} \mathrm{C}$. Part I, Proc. Am. Acad. Arts Sci. 68, 505-564 (1933).

[12] Barieau, R. E., and Dalton, B. J., A method for treating PVT data from a Burnett compressibility apparatus, Bureau of Mines Report of Investigations 7020 (1967).

[13] Hall, K. R., and Canfield, F. B., A least-squares method for reduction of Burnett data to compressibility factors and virial coefficients, Physica 47,99-108 (1970).

[14] Roark, R. J., Formulas for Stress and Strain (McGraw-Hill Book Company, Inc., New York, 1954).

[15] Kadkowski, P. P., Bluhm, J. I., and Bowie, O. L., Watertown Arsenal Laboratory, Thick-Walled Cylinder Handbook.

[16] Briggs, T. C.. Dalton, B. J., and Barieau, R. E., Compressibility data for helium at $0{ }^{\circ} \mathrm{C}$ and pressures to 800 atmospheres, Bureau of Mines Report of Investigations 7287 (1969).

[17] Marquardt, D. W., An algorithm for least-squares estimation of nonlinear parameters, J. Soc. Indust. Appl. Math. 1 1, 431-441 (1963).

[18] Michels, A., Wijker, Hub., and Wijker, Hk., Isotherms of argon between $0{ }^{\circ} \mathrm{C}$ and $150{ }^{\circ} \mathrm{C}$ and pressures up to 2900 atmospheres, Physica 15, 627-633 (1949).

[19] Levelt Sengers, J. M. H., Klein, M., and Gallagher, J. S., Pressure-Volume-Temperature relationships of gases. Virial coefficients, American Institute of Physics Handbook, (to be published).

[20] Butcher, E. G., and Dadson, R. S., The virial coefficients of the carbon dioxide-ethylene system I. Pure gases, Proc. Roy. Soc. (London) A2 77 , 448-467 (1964).

[21] Dadson, R. S., Evans, E. J., and King, J. H., The second virial coefficient of carbon dioxide, Proc. Phys. Soc. 92, 1115-1121 (1967).

[22] Brunauer, S., The Adsorption of Gases and Vapors. Volume I Physical Adsorption (Princeton University Press, Princeton, 1943).

(Paper 75C3\&4-323) 